\title{
Correction to: Acoustics-A Textbook for Engineers and Physicists
}

\section{Correction to:}

\section{J. H. Ginsberg, Acoustics-A Textbook}

for Engineers and Physicists, https://doi.org/10.1007/978-3-319-56847-8

The original version of the book was inadvertently published without including the Electronic Supplementary Material files in Chaps. 7-13.

The erratum book has been updated with the changes. 